\title{
ESTIMATION OF COSMOLOGICAL CONSTANT OF NEARBY SPIRAL GALAXIES USING ROTATION CURVE
}

\author{
R. K. Bachhan*, B. Aryal* and U. Khanal* \\ *Central Department of Physics, Tribhuvan University, Kirtipur, Nepal
}

\begin{abstract}
An expression for rotational velocity of a test particle around the central mass in the invariant plane is derived. For this, a line element of Schwarzschild de-Sitter space-time is used to study the effect of cosmological constant $\Lambda$ ) on the motion of both massive and mass-less particles in the Universe. This expression is fitted for the mass and $\Lambda$. For this, the total mass $M$ is divided into the mass of the black hole $M_{B}$ and a uniform sphere of radius $r$ dominated by dust $\left(\rho_{d}\right)$ which is supposed to vary as $\rho_{d}=\frac{\rho_{0}}{r}$, i.e., $M=M_{B}+4 \pi \int_{0}^{r} \rho_{d} r^{2} d r$. Using rotation curve data of 6 nearby spiral and barred spiral galaxies, we estimated the value of $\Lambda$ and the mass of the galaxy. The cosmological constant $(\Lambda)$ is found to be positive for all 6 galaxies. The positive $\Lambda$ reveals the importance of de Sitter space, suggesting accelerating Universe. The cosmological constant $(\Lambda)$ and the density $\left(\rho_{0}\right)$ are found to lie in the range $1.269 \times 10^{-41}$ to $9.490 \times 10^{-42} \mathrm{~km}^{-2}$, and $7.044 \times 10^{-9}$ to $3.250 \times 10^{-8}$ $\mathrm{km}^{-1}$, respectively. The mass $M_{B}$ is found in the range $1.867 \times 10^{7}$ to $1.730 \times 10^{10}$ solar mass. Our result shows a good agreement with the results of supernova project which gives positive $\Lambda$ value, suggesting accelerating Universe.
\end{abstract}

Keywords: Galaxies formation; General astronomical databases.

\section{INTRODUCTION}

The cosmological constant $(\Lambda)$ is a parameter describing the energy density of the vacuum (empty space) ${ }^{1}$. A negative $\Lambda$ adds to the attractive gravity of matter whereas a positive $\Lambda$ resists the attractive gravity of matter due to its negative pressure $^{1}$. Two supernova cosmology project converge on a common result: cosmos will expand forever at an accelerating rate, pushed on by dark energy $(\Lambda)^{2}$. According to this, the Universe is discovered to have a flat spatial geometry $(k=0)$, a positive cosmological constant (dark energy) $\Omega_{\Lambda}=0.7$, and matter density (of all kinds) $\Omega_{\mathrm{M}}=0.7^{3}$. The data from the Boomerang experiment provide a firm evidence for the case of flat Universe $\Omega_{\mathrm{k}} \sim 0{ }^{2,4}$ discussed some of the theoretical justifications for non zero $\Lambda^{4}$.

Kraniotis \& Whitehouse (2000) interpreted $\Lambda$ as the nongravitational contribution to galactic velocity rotation curves and estimated negative $\Lambda$ for 6 spiral galaxies, suggesting deaccelerating universe $\mathrm{e}^{5}$. The implications of this result will be discussed later.

In the present work, we discuss an expression for rotational velocity of a test particle in a circular motion around the central mass in an asymptotically de Sitter space time ${ }^{6}$. This expression is used to estimate the value of the $\Lambda$ from the rotation curve of various spiral galaxies. We estimated the value of $\Lambda$ for 6 galaxies that have radial velocity less than $5,000 \mathrm{~km} / \mathrm{s}$.
This paper is organized as follows. A relation between rotational velocity and the cosmological constant is derived in Sect. 2. We describe the database and the calculation procedure in Sects. 4. In Sect. 5, we present our result. Finally, a discussion of results and the conclusions are summarized in Sect. 5.

\section{THEORY}

The metric which describes the geometry of the Schwarzschild de-Sitter space-time is

$d s^{2}=\left(1-\frac{2 M}{r}-\frac{\Lambda r^{2}}{3}\right) d t^{2}-\frac{d r^{2}}{\left(1-\frac{2 M}{r}-\frac{\Lambda r^{2}}{3}\right)}-r^{2}\left(d \theta^{2}+\sin ^{2} \theta d \varphi^{2}\right)$

Here we utilize this line element in order to study the effect of the cosmological constant $\ddot{E}$ on the motion of both massive and massless particles in the Universe. For this space-time, the Lagrangian is

$\mathcal{L}=\frac{1}{2}\left[\left(1-\frac{2 M}{r}-\frac{\Lambda r^{2}}{3}\right) \dot{t}^{2}-\frac{\dot{r}^{2}}{\left(1-\frac{2 M}{r}-\frac{\Lambda r^{2}}{3}\right)}-r^{2} \dot{\theta}^{2}-r^{2} \sin ^{2} \theta \dot{\varphi}^{2}\right]$

where dot represents differentiation with respect to the affne parameter t. The canonical momenta are

$$
\begin{aligned}
& \wp_{t}=\frac{\partial \mathcal{L}}{\partial \dot{t}}=\left(1-\frac{2 M}{r}-\frac{\Lambda \mathrm{r}^{2}}{3}\right) \dot{t} \\
& \wp_{\theta}=-\frac{\partial \mathcal{L}}{\partial \dot{\theta}}=r^{2} \dot{\theta} \\
& \wp_{r}=-\frac{\partial \mathcal{L}}{\partial \dot{r}}=\left(1-\frac{2 M}{r}-\frac{\Lambda \mathrm{r}^{2}}{3}\right)^{-1} \dot{r}
\end{aligned}
$$

Author for Correspondence: R. K. Bachhan, Central Department of Physics, Tribhuvan University, Kirtipur, Nepal. 
and

$\wp_{\varphi}=-\frac{\partial \mathcal{L}}{\partial \dot{\varphi}}=r^{2} \sin ^{2} \theta \dot{\varphi}$

The resulting Hamiltonian is

$\mathcal{H}=\wp_{t} \dot{t}-\left(\wp_{r} \dot{r}+\wp_{\theta} \dot{\theta}+\wp_{\varphi} \dot{\varphi}\right)-\mathcal{L}=\mathcal{L}$

The equality of Hamiltonian and Lagrangian signifies that there is no 'potential' in the problem. Thus, the energy is derived solely from 'kinetic energy'. Now we rescale the affine parameter $t$ in such away that the $2 \mathrm{~L}$ has the value +1 for time-like geodesics and zero for null geodesics. In this case the integrals of motion in the invariant plane $\theta=\frac{\pi}{2}$ are

$\wp_{t}=\left(1-\frac{2 M}{r}-\frac{\Lambda \mathrm{r}^{2}}{3}\right) \dot{t}=E$

and

$\wp_{\varphi}=r^{2} \dot{\varphi}=L$

where $\mathrm{E}$ and $\mathrm{L}$ are constants associated with the energy and angular momentum of the particle (about an axis normal to the invariant plane) respectively. Using and (equations 8 and 9), the constancy of the Lagrangian gives (Chandrashekhar 1983) ${ }^{7}$

$\frac{E^{2}}{\left(1-\frac{2 M}{r}-\frac{\Lambda \mathrm{r}^{2}}{3}\right)}-\frac{\dot{r}^{2}}{\left(1-\frac{2 M}{r}-\frac{\Lambda \mathrm{r}^{2}}{3}\right)}-\frac{L^{2}}{r^{2}}=2 \mathcal{L}=+1$ or 0

depending upon whether we are considering time-like or null geodesics. Defining further a parameter W, where,

$\Omega=\frac{d \varphi}{d t}=\frac{\dot{\varphi}}{\dot{t}}=\frac{L}{E}\left[\frac{\left(1-\frac{2 M}{r}-\frac{\Lambda \mathrm{r}^{2}}{3}\right)}{r^{2}}\right]$

The rotational velocity of a test particle around the central mass in the invariant plane is given by, $v_{\varphi}=\frac{r \Omega}{\sqrt{1-\frac{2 M}{r}-\frac{\Lambda \mathrm{r}^{2}}{3}}}$ (12)

For time-like geodesics, equations (10) and (11) can be rewritten in the form

$\left(\frac{d r}{d \tau}\right)^{2}+\left(1-\frac{2 M}{r}-\frac{\Lambda r^{2}}{3}\right)\left(1+\frac{L^{2}}{r^{2}}\right)=E^{2}$

and

$\left(\frac{d \varphi}{d \tau}\right)^{2}=\frac{L}{r^{2}}$

Dividing equation (13) by the $\left(\frac{d \varphi}{d \tau}\right)^{2}$ from equation (14), we can get the equation for $r$ as a function of ö:

$\left(\frac{d r}{d \varphi}\right)^{2}=\frac{\Lambda r^{6}}{3 L^{2}}+\left(E^{3}+\frac{\Lambda L^{2}}{3}-1\right) \frac{r^{4}}{L^{2}}+\frac{2 M r^{3}}{L^{2}}-r^{2}+2 M r$

Now letting ,we obtain the basic equation of the problem

$$
\left(\frac{d u}{d \varphi}\right)^{2}=2 M u^{3}-u^{2}+\frac{2 M u}{L^{2}}+\frac{\Lambda}{3 L^{2} u^{2}}-\left(\frac{1-E^{2}}{L^{2}}-\frac{\Lambda}{3}\right)=f(u)
$$

Since $f(u)$ involves a polynomial of fifth degree (i.e., quantic polynomial). The exact solution to the problem cannot be formulated in terms of elliptic integrals ${ }^{8,9}$. For circular orbits, $f(r)$ in equation (13) should have cauldeut root. The critical values of $\mathrm{L}$ and $\mathrm{E}$ required for such cauldeut roots can be found, by setting $f(r)$ and $f^{\prime}(r)$ equal to zero. Accordingly,

$f(r)=\dot{r}^{2}=E^{2}-\left(1-\frac{2 M}{r}-\frac{\Lambda r^{2}}{3}\right)\left(1+\frac{L^{2}}{r^{2}}\right)=0$

and

$f^{\prime}(r)=\frac{d f}{d r}=-\left(1-\frac{2 M}{r}-\frac{\Lambda \mathrm{r}^{2}}{3}\right)\left(-\frac{2 L^{2}}{r^{3}}\right)-\left(1+\frac{L^{2}}{r^{2}}\right)\left(\frac{2 M}{r^{2}}-\frac{2 \Lambda \mathrm{r}}{3}\right)=0$

Eliminating the factor $\left(\left(1+\frac{L^{2}}{r^{2}}\right)\right)$, we get

$\frac{L}{E}=\frac{r \sqrt{\frac{M}{r}-\frac{\Lambda \mathrm{r}^{2}}{3}}}{1-\frac{2 M}{r}-\frac{\Lambda \mathrm{r}^{2}}{3}}$

Equation (11) reduces to

$\Omega=\frac{\sqrt{\frac{M}{r}-\frac{\Lambda \mathrm{r}^{2}}{3}}}{r}$

By substituting equation (20) in equation (12), we find

$v_{\varphi}=\sqrt{\frac{\frac{M}{r}-\frac{\Lambda \mathrm{r}^{2}}{3}}{1-\frac{2 M}{r}-\frac{\Lambda \mathrm{r}^{2}}{3}}}$

This equation represents the rotational velocity of test particle around the central mass in the invariant plane. This expression can be used to determine the mass and the cosmological constant (ت̈) of galaxies using rotation curve data.

At the centre of the galaxy there lies a massive black hole. We suspect that the black hole has a prominent role in describing the shape and size of the galaxy. We also suspect that a central black hole might affect the value of cosmological constant (Ë). We disintegrate the total mass $M$ into the mass of the black hole $M_{B}$ and a uniform sphere of radius r dominated by dust $\left(\rho_{d}\right)$ which we supposed to vary as $\rho_{d}=\frac{\rho_{0}}{r}$, i.e., we write $M=M_{B}+4 \pi \int_{0}^{r} \rho_{d} r^{2} d r$ and get

$\frac{v}{c}=\sqrt{\left(\frac{\frac{M_{B}}{r}+2 \pi \rho_{0} r-\frac{\Lambda r^{2}}{3}}{1-\frac{2 M_{B}}{r}-4 \pi \rho_{0} r-\frac{\Lambda r^{2}}{3}}\right)}$

We fit the relation (22) to calculate the value of $M_{B}, \Lambda$ and $\rho_{0}$.

\section{DATABASE}

We have studied rotation curves of 6 galaxies. The observed database of these rotation curves are provided by Sofue ${ }^{10,11}$. The rotation curves (dashed line) and the images of the 
Table 1: First three columns list the name and position of the galaxy. The Hubble recessional velocity is given in the column 4 . Fifth column shows the morphology of galaxies. The last two columns list the major and minor diameters of our galaxies. [source: NASA Extragalactic Database]

\begin{tabular}{|c|c|c|c|c|c|c|}
\hline Name & RA (deg.) & Dec (deg.) & RV (km/s) & Morphology & a (arc min) & b (arc min) \\
\hline NGC 0598 & 22.7583 & 30.4042 & -179 & Sc & 70.8 & 41.7 \\
\hline NGC 1097 & 41.0458 & -30.4836 & 1271 & SBb & 9.3 & 6.3 \\
\hline NGC 1417 & 54.8667 & -04.8639 & 4114 & Sb & 2.7 & 1.7 \\
\hline NGC 2590 & 125.6208 & -00.4283 & 4996 & Sb & 2.2 & 0.7 \\
\hline NGC 2708 & 133.4042 & -03.1681 & 2008 & Sb & 2.6 & 1.3 \\
\hline NGC 3198 & 154.2167 & 45.8017 & 663 & SBb & 8.5 & 3.3 \\
\hline
\end{tabular}

galaxies are shown in Fig. 2. The rotation curves of these galaxies comprise steep central rise, bulge component, broad maximum by the disk and the halo component ${ }^{10}$. It can be seen that the rotational velocities in many well resolved galaxies do not declined to zero at the nucleus ${ }^{10}$. This hints that the mass density increases rapidly towards the nucleus than the expected from exponential or de Vaucouleurs laws ${ }^{12}$. The widely adopted zero velocity at the center is due to the linkage (merely a drawing) between positive and negative sides of the nucleus ${ }^{12}$. However, we focus our attention in the flat region of the rotation curve. The basic properties of these galaxies are listed in Table1.

We use the value of $R$ (in $\mathrm{kpc}$ ) and $V$ (in $\mathrm{km} \mathrm{s}^{-1}$ ) from the given rotation curves in order to estimate the value of the mass (M) and cosmological constant $(\Lambda)$ of galaxies.

\section{RESULT \& DISCUSSION}

A typical sketch of the galaxy rotation curve is shown in Fig. 1. The extreme flattened point of the galaxy rotation curve is the point where the surface brightness of the galaxy suddenly falls. The radius $r$ represents the semi major diameter of the galaxy.

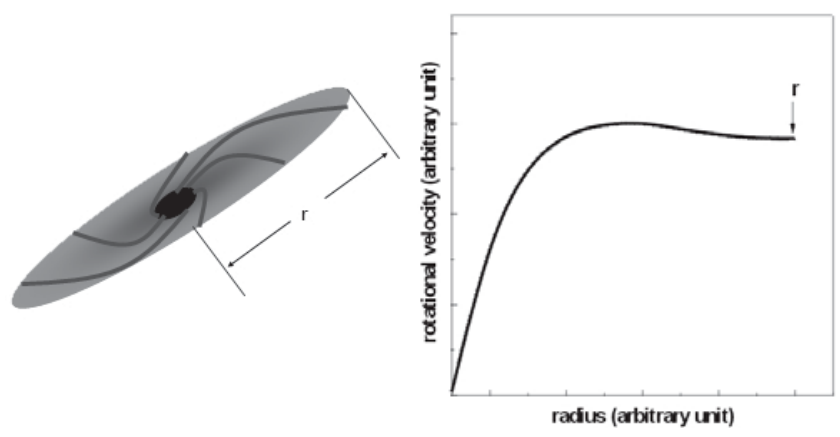

Figure 1: A typical sketch of the galaxy and its rotation curve.

Table 2 shows the values of the mass $(\mathrm{M})$ and the cosmological constant $(\Lambda)$ of 6 sample galaxies. The cosmological constant $(\Lambda)$ is found to be positive suggesting accelerating universe.

Table 2: The first column lists the NGC name of the galaxy. The second column gives the mass of the galaxy. The last two columns give the cosmological constant and the density.

\begin{tabular}{|l|c|c|c|}
\hline Name & $\mathbf{M}_{\mathbf{B}}$ (solar mass) & $\left.\boldsymbol{\Lambda} \mathbf{( k m}^{-2}\right)$ & $\rho_{\mathbf{o}}\left(\mathbf{k m}^{-1}\right)$ \\
\hline NGC 0598 & $1.867 \times 10^{7}$ & $1.269 \times 10^{-41}$ & $7.044 \times 10^{-9}$ \\
\hline NGC 1097 & $1.730 \times 10^{10}$ & $9.390 \times 10^{-42}$ & $1.788 \times 10^{-8}$ \\
\hline NGC 1417 & $2.486 \times 10^{8}$ & $8.116 \times 10^{-42}$ & $2.982 \times 10^{-8}$ \\
\hline NGC 2590 & $6.380 \times 10^{9}$ & $3.873 \times 10^{-41}$ & $2.329 \times 10^{-8}$ \\
\hline NGC 2708 & $2.106 \times 10^{8}$ & $4.156 \times 10^{-41}$ & $3.250 \times 10^{-8}$ \\
\hline NGC 3198 & $1.823 \times 10^{9}$ & $3.119 \times 10^{-42}$ & $5.835 \times 10^{-9}$ \\
\hline
\end{tabular}

The value of the mass $M_{B}$ is found in the range $1.867 \times 10^{7}$ to $1.730 \times 10^{10}$ solar mass. The cosmological constant $(\Lambda)$ and the density $\left(\rho_{\mathrm{o}}\right)$ are found to lie in the range $1.269 \times 10^{-41}$ to $9.490 \times 10^{-42} \mathrm{~km}^{-2}$, and $7.044 \times 10^{-9}$ to $3.250 \times 10^{-8} \mathrm{~km}^{-1}$, respectively. Fig. 2 shows the fitted rotation curves using our model as described in equation (22). The dashed curves represent the observed values of the rotation curve. The images of the galaxies can be seen in each plot. The dark thick curve represents our model in which we assumed $\left(\boldsymbol{\rho}_{\text {clust }}\right)$ $=\frac{\rho_{0}}{r}$, i.e., $M=M_{B}+4 \pi \int_{0}^{r} \rho_{d} r^{2} d r$ where, $M$ into the mass of the black hole $M_{B}$ and a uniform sphere of radius $r$ dominated by dust $\left(\rho_{\text {clust }}\right)$.
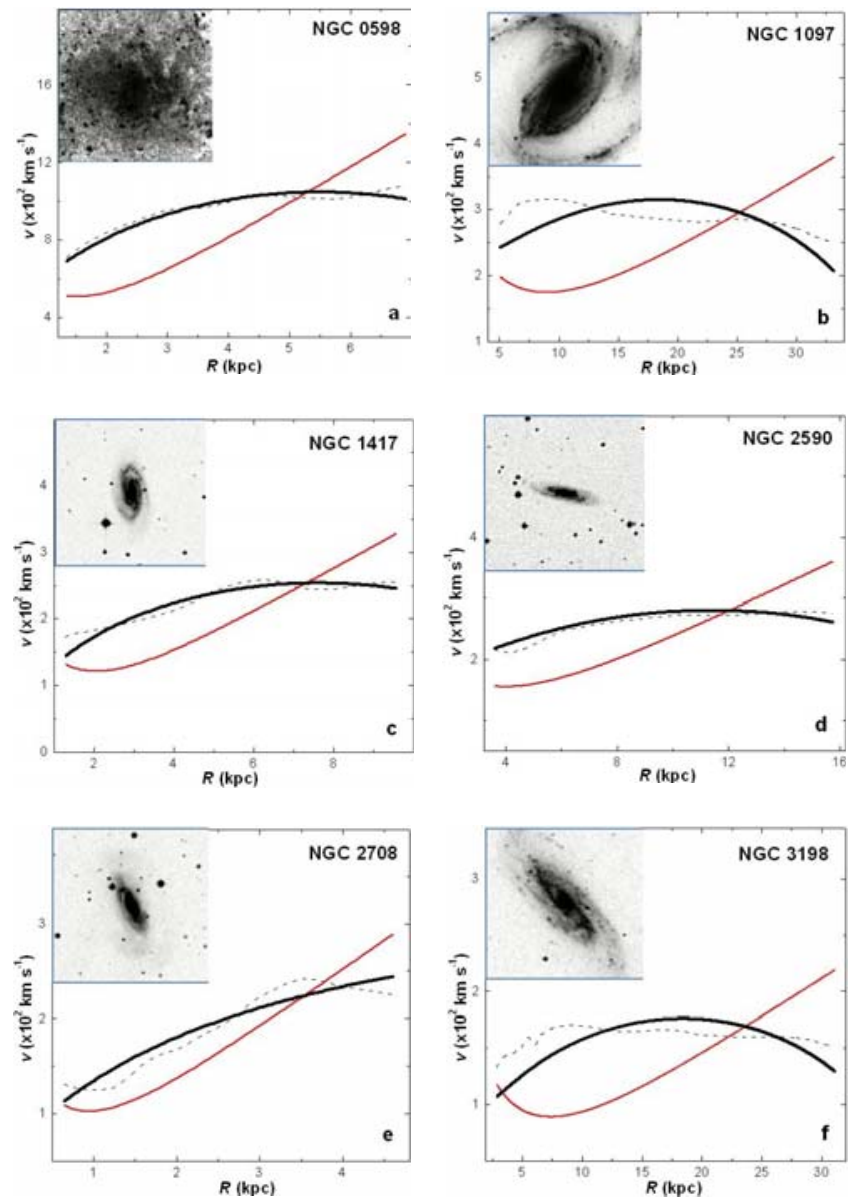

Figure 2: Observed galaxy rotation curve ${ }^{11}$. The black thick line represents our model. A good agreement between our model and the observed distribution (dashed lines) can be seen. The third line is the old model in which the mass function is assumed to be constant through out the galaxy. The value of cosmological constant is found to be negative assuming this old model. 
Kraniotis \& Whitehouse (2000) estimated $\Lambda$ value for 6 spiral galaxies using Friedman energy equation ${ }^{12,13}$, weak field approximation (Whitehouse \& Kraniotis 1999), and the observed rotation curve of the galaxy ${ }^{13,14}$. They found that the rotation curve data of all 6 galaxies up $50 \mathrm{kpc}$ (see Table 1 of their paper) inevitably lead to a negative value of the cosmological constant, suggesting de-accelerating Universe.

The result obtained by Kraniotis \& Whitehouse (2000) contradicts the result obtained from the Supernova Cosmology project ${ }^{14}$. It has to be kept in mind that the Supernova project studied the recessional velocity of distant objects while in this paper, we are concerned with the rotational velocity of galaxies. Obviously, the bound matter in galaxies can not be accelerating with the expansion of the universe. So during the formation of galaxies local effect must have worked in such away that, an effect of negative vacuum energy was generated in the region. Probably, the vacuum energy of these structures leads to form a nearby local bubble.

\section{CONCLUSION}

We used an expression for rotational velocity of a test particle in a circular motion around the central mass in an asymptotically de Sitter space time and estimated the value of the $\Lambda$ from the rotation curve data of 6 galaxies $^{6}$. We conclude the following:

(1) The cosmological constant $(\Lambda)$ is found to be positive for all 6 galaxies. Their values lie in the range $1.269 \times 10^{-41}$ to 9.490 $\mathrm{x} 10^{-42} \mathrm{~km}^{-2}$.

(2) The total mass $M$ is divided into the mass of the black hole and a uniform sphere of radius $r$ dominated by $\left(\rho_{d}\right)$ dust which is supposed to vary as $\rho_{d}=\frac{\rho_{0}}{r}$, i.e., $M=M_{B}+4 \pi \int_{0}^{r} \rho_{d} r^{2} d r$. The mass $M_{B}$ is found in the range 1.867 $\mathrm{x} 10^{7}$ to $1.730 \times 10^{10}$ solar mass. The density $\left(\rho_{0}\right)$ is found to lie in the range $7.044 \times 10^{-9}$ to $3.250 \times 10^{-8} \mathrm{~km}^{-1}$.

(3) Our results show that the relative effect of cosmological constant $(\Lambda)$ becomes pronounced at larger distances, away from the bulge of the galaxy.
(4) A similar result is noticed for a blue-shifted galaxy, NGC0598.

The result of this work is in good agreement with the results of supernova project which looked at the large scale cosmology. We intend to justify this by analysing rotation curves of more galaxies in our future works.

\section{ACKNOWLEDGEMENTS}

This research has made use of the NASA/IPAC Extragalactic Database (NED) which is operated by the Jet Propulsion Laboratory, California Institute of Technology, under contract with the National Aeronautics and Space Administration. One of the authors (RKB) acknowledges Central Department of Physics, Tribhuvan University, Kirtipur, for providing various support for his masters (M.Sc.) thesis.

\section{REFERENCES}

1. Sancisi R., van Albada T. S. 1987, in IAU Symp. 117, Dark matter in the Universe, ed. J. Kormemdy \& G. R. Knapp (Dordiecht: Reidel), 67.

2. Perlmutter S. et al. 1999. Astro-ph/9812133

3. Perlmutter S. et al. 2003, Physics Today, p81

4. Sanders R. H. 1996, ApJ, 473, 117

5. Kraniotis G. V., Whitehouse S. V., 2000, astro-ph/0002391

6. Pokheral R. 1994, M. Sc. Thesis, Tribhuvan Univ., Kirtipur, Nepal

7. Chandrashekhar S. 1983, The Mathematical Theory of Black Holes, Oxford Univ. press

8. Gradshteyn I. S., Ryzhik I. M., Table of Integrals, Series and Products, Academic press, 5th ed., 1994

9. Goldstein S. 1982, Classical Mechanics, Narosa Publishing

10. Sofue Y. et al 1999, ApJ, 523, 136

11. Sofue Y. 2008, private communication

12. Sciama D. 1995, Modern Cosmology and the Dark Energy Problem, Cambridge University press

13. Bahcall N., Ostriker J., Perlmutter S., Steinhardt S., 1999, astro$\mathrm{ph} / 9906463$

14. Whitehouse S. V., Kraniotis G. V., 1999, astro-ph/9911485. 